\title{
A FAMILY OF ABELIAN VARIETIES RATIONALLY ISOGENOUS TO NO JACOBIAN
}

\author{
JAMES L. PARISH
}

(Communicated by Louis J. Ratliff, Jr.)

\begin{abstract}
Let $E_{d}$, for any $d \in \mathbf{Q}(i)^{*}$, be the curve $x^{3}-d x z^{2}=y^{2} z$, and let $g$ be any positive integer. It is shown that, if $d$ is not a square in $\mathbf{Q}(i)$ and $g>1$, the abelian variety $E_{d}^{g}$ is not isogenous over $\mathbf{Q}(i)$ to the Jacobian of any genus- $g$ curve. The proof proceeds by showing that any curve whose Jacobian is isogenous to $E_{d}^{g}$ over $\mathbf{Q}(i)$ must be hyperelliptic, and then showing that no hyperelliptic curve can have Jacobian isogenous to $E_{d}^{g}$ over $\mathbf{Q}(i)$.
\end{abstract}

\section{INTRODUCTION}

One of the great delights of algebraic geometry is the interplay between the arithmetic and the geometry of a situation. The choice of a field of definition for a problem frequently has interesting effects on its solution. In this paper, a rather unusual example of this interplay is presented. Let $d \in \mathbf{Q}(i)^{*}$. Define $E_{d}$ to be the plane curve whose equation is $x^{3}-d x z^{2}=y^{2} z . E_{d}$ is defined over $\mathbf{Q}(i)$. It is an elliptic curve, with complex multiplication by the ring $\mathbf{Z}[i]$, and this complex multiplication is likewise defined over $\mathbf{Q}(i)$. It is of interest to consider which curves of given genus $g$, defined over $\mathbf{Q}(i)$, have Jacobian varieties isogenous over $\mathbf{Q}(i)$ to $E_{d}^{g}$. It is known (Bloch [1]) that the Jacobian of the curve $x^{4}+y^{4}+z^{4}=0$ is isogenous to $E_{1}^{3}$ over $\mathbf{Q}(i, \sqrt{2})$; thus the following result is somewhat surprising.

Theorem 1. If $d$ is not a square in $\mathbf{Q}(i)$ and $g>1$, no curve of genus $g$ has Jacobian isogenous to $E_{d}^{g}$ over $\mathbf{Q}(i)$.

This theorem follows easily from the next.

Theorem 2. Let $C$ be a curve of genus $g$, rational over $\mathbf{Q}(i)$. Suppose that, for some $d$ not a square in $\mathbf{Q}(i)$, the Jacobian variety $J(C)$ is isogenous to $E_{d}^{g}$ over $\mathbf{Q}(i)$. Then $C$ is hyperelliptic.

Coupling this result with some standard facts about holomorphic differentials on hyperelliptic curves yields Theorem 1 .

Received by the editors June 10, 1987 and, in revised form, March 2, 1988.

1980 Mathematics Subject Classification (1985 Revision). Primary 14K15. 
The idea of the proof of Theorem 2 is as follows. First, Weil's reformulation [7] of the Torelli theorem is quoted, and it is shown that, if the Jacobian $(J(C), \theta)$ with its canonical principal polarization has an automorphism whose square is the inversion map $l$, then $C$ is hyperelliptic. Next, it is shown that, for $d$ nonsquare in $\mathbf{Q}(i)$, any Abelian variety isogenous over $\mathbf{Q}(i)$ to $E_{d}^{g}$ inherits the latter's multiplication by $i$. Finally, it is shown that any principal polarization on such an Abelian variety is stable under the inherited automorphism $i$. The result is then immediate.

The inspiration for this work rests in cycle theory. Two decades ago Griffiths [3] discovered that homological and algebraic equivalence of cycles on a variety are not necessarily identical. Since then, considerable effort has gone into investigating the difference between the two. Work has proceeded on two fronts. Some researchers-notably Griffiths, Ceresa, and Clemens-have studied the question over C; others, including Bloch, Swinnerton-Dyer, and B. Harris, have considered more arithmetic aspects, studying cycles which are rational over a given number field.

For the purposes of this paper, the most interesting result by the first group of researchers is as follows. Let $C$ be a nonsingular genus $-g$ curve, $J$ its Jacobian, and $l: J \rightarrow J$ the inversion map. Over $\mathbf{C}$ we have the Abel-Jacobi map imbedding $C$ in $J$, and a cycle $\Xi=C-{ }_{l} C$ may be defined. $\Xi$ is homologically trivial, because $l$ acts trivially on, say, de Rham cohomology. If $C$ is hyperelliptic, $l C$ is a translate of $C$ and hence $\Xi$ is algebraically trivial. However, Ceresa [2] has shown that, if $g \geq 3$, for $C$ a sufficiently general genus $-g$ curve, $n \Xi$ is algebraically nontrivial for all $n \in \mathbf{Z}^{+}$. Define $\operatorname{Griff}(A)$, for any Abelian variety $A$, to be the group of homologically trivial dimension-one cycles on $A$ modulo algebraic equivalence; this says that $\Xi$ is a nontorsion element of $\operatorname{Griff}(J)$. Furthermore, if $A$ is another Abelian variety and $\phi: A \rightarrow J$ is an isogeny, then $\phi^{*}(\Xi) \in \operatorname{Griff}(A)$ is likewise of infinite order, by the projection formula. This suggests that one way to find nontrivial elements of $\operatorname{Griff}(A)$ is to construct isogenies from $A$ to various Jacobian varieties.

Now restrict attention to the group $\operatorname{Griff}(A, k)$ of cycles, rational over a field of definition $k$, which are homologically trivial, modulo algebraic equivalence. (Note that $\Xi$ may not be $k$-rational even if $C$ is, as, if $C(k)=\varnothing$, the Abel-Jacobi map is not $k$-rational.) The second group of researchers has been examining an analogue of the Birch-Swinnerton-Dyer conjecture. This analogue claims that the rank of $\operatorname{Griff}(A, k)$ equals the order of vanishing at $s=g-1$ of the $L$-function $L(M, s)$, where $M$ is a certain submotive of $H^{2 g-2}(A)$. For details, see Bloch [1]. In that paper, Bloch constructs an infinite-order element of $\operatorname{Griff}\left(E_{1} \times E_{1} \times E_{-1}, \mathbf{Q}\right)$ by displaying an isogeny to the Jacobian of the Fermat quartic $x^{4}+y^{4}=z^{4}$. It is not difficult to adjust this to give a $\mathbf{Q}(i, \sqrt{2})$-rational isogeny from $E_{1}^{3}$ to the Jacobian of $x^{4}+y^{4}+z^{4}=0$, as mentioned above. Now, Stephens [7] has shown that, for certain values of $d$, 
the aforementioned $L$-function for $E_{d}^{3}$ vanishes to order at least two. Coupled with the conjecture, this suggests (rather weakly) that there may be, so to speak, "extra" isogenies to Jacobians for these values of $d$. More specifically, it suggests a search for Q-rational isogenies from $E_{d}^{3}$ to Jacobians. The import of Theorem 1 is that this particular task is fruitless; no such isogenies exist for nonsquare $d$. (It is, of course, possible that isogenies from $E_{d}^{3}$ to Jacobians which are not $\mathbf{Q}(i)$-rational can give rise to nontorsion cycles in $\operatorname{Griff}\left(E_{d}^{3}, \mathbf{Q}\right)$ via a norm process; this is not considered here.)

\section{JACOBIAN VARIETIES WITH MULTIPLICATION BY $i$}

We begin with Weil's version of the Torelli theorem.

Theorem 3 (Weil). Let $C$ be a nonsingular curve and $(J, \Theta)$ its Jacobian with its canonical polarization. Then $\operatorname{Aut}(C)$ injects into $\operatorname{Aut}(J, \theta)$. If $C$ is not hyperelliptic, the image has index two and does not contain the inversion map $l$. Otherwise the map is onto, and $l$ is the image of the hyperelliptic involution.

Proof. See [8].

Corollary. Suppose the Jacobian $J$ of $C$ has an automorphism whose square is the inversion map and which preserves $\boldsymbol{\Theta}$. Then $C$ is hyperelliptic.

Proof. Since $\operatorname{Aut}(C)$ has index $\leq 2$ in $\operatorname{Aut}(J, \Theta)$, the square of any element of $\operatorname{Aut}(J, \theta)$ lies in $\operatorname{Aut}(C)$. But $l$ is the square of such an automorphism and hence is in $\operatorname{Aut}(C)$. It follows that $C$ is hyperelliptic.

\section{RATIONALITY OF FINITE SUBGROUPS OF $E_{d}^{g}$}

In this section we prove a general result concerning the rationality of finite subgroups of elliptic curves with complex multiplication. It will follow from this result that $\mathbf{Q}(i)$-rational subgroups of $E_{d}^{g}$ with odd order are necessarily stable under $i$, and if $d$ is not a square in $\mathbf{Q}(i)$ the same is true of even-order subgroups. (N.B. Much more than this can be proven, but the computations are tedious. For present purposes, this result will suffice.) This implies the inheritance of $i$ by Abelian varieties isogenous to $E_{d}^{g}$ over $\mathbf{Q}(i)$ for nonsquare $d$, as stated in the introduction.

Let $F$ be a quadratic imaginary extension of $\mathbf{Q}$, and let $H$ be its Hilbert class field, i.e., its maximal abelian unramified extension. Let $E$ be an elliptic curve, rational over $H$, with complex multiplication (over $H$ ) by the integers $O$ of $F$. ( $H$ is the smallest field over which this is possible; for this and the following, see Gross [4].) For any positive integer $m$, let $H_{m}$ be the field of definition of the $m$-torsion points of $E . H_{m}$ is Galois over $H$; let $G_{m}$ be the Galois group. Let $H^{\prime}$ be the union of all the fields $H_{m}$, and $G=\operatorname{Gal}\left(H^{\prime} / H\right)$. Let $\mu_{m}$ be the group of the $m$ th roots of unity, and let $\alpha \in F$ be such that $O=\mathbf{Z}[\alpha]$. 
The group ${ }_{m} E$ of $m$-torsion points on $E$ is isomorphic to $(\mathbf{Z} / m)^{2}$. The action of $G_{m}$ on ${ }_{m} E$ therefore induces an injection $G_{m} \rightarrow G L_{2}(\mathbf{Z} / m)$. Likewise, the units $O^{*}$ of $O / m O$ act on ${ }_{m} E$ and we have an injection $(O / m O)^{*} \rightarrow$ $G L_{2}(\mathrm{Z} / m)$. Since the endomorphisms of $E$ are defined over $H, G_{m}$ commutes with them. It easily follows that the image of $G_{m}$ is in the image of $(O / m O)^{*}$. We will systematically confuse $G_{m}$ with its image in $(O / m O)^{*}$.

It follows that, allowing $O$ to act diagonally on $E^{g}$ for any $g$, any finite subgroup of $E^{g}$ which is stable under $O$ is rational over $H$. The converse is not precisely true, but very nearly so, as we shall see. For a finite subgroup of $E^{g}$, rationality over $H$ is equivalent to the same property for its Sylow subgroups. Thus, we can restrict attention to subgroups of prime power order. Let $p$ be a fixed prime number, and consider the maps $G_{p^{k}} \rightarrow\left(O / p^{k} O\right)^{*}$ for various $k$. These give rise to a map $G \rightarrow\left(O \otimes \mathbf{Z}_{p}\right)^{*}$, and we let $R_{p}$ be the $\mathbf{Z}_{p}$-algebra generated by the image of $G$. Clearly, rationality of a $p$-group is equivalent to stability under $R_{p}$. We turn to the consideration of this ring.

Lemma. The $\mathbf{Z}_{p}$-subalgebras of $O \otimes \mathbf{Z}_{p}$ are $\mathbf{Z}_{p}$ itself and the rings $\mathbf{Z}_{p}\left[p^{k} \alpha\right]$, $k=0,1,2, \ldots$.

Proof. Let $R \subseteq O \otimes \mathbf{Z}_{p}$ be a $\mathbf{Z}_{p}$-algebra. Considered as a $\mathbf{Z}_{p}$-module, $R$ is finitely-generated and torsion-free; hence it is free of rank $\leq 2$. If $R$ has rank one, then, since it contains $\mathbf{Z}_{p}$, it is $\mathbf{Z}_{p}$ itself. Otherwise $R$ has a free basis of the form $\{1, \beta\}$. Write $\beta=\beta^{\prime}+p^{k} \mu \alpha$ where $\beta^{\prime} \in \mathbf{Z}_{p}, k \geq 0$, and $\mu \in \mathbf{Z}_{p}^{*}$. Then $\left\{1, p^{k} \alpha\right\}$ is also a free basis for $R$, and the result follows.

Corollary. If $G_{p} \nsubseteq(\mathbf{Z} / p)^{*}$, then $R_{p}=\mathbf{Z}_{p}[\alpha]$.

Proof. $G_{p} \subseteq R_{p} /\left[p\left(O \otimes \mathbf{Z}_{p}\right) \cap R_{p}\right]$. If $R_{p} \neq \mathbf{Z}_{p}[\alpha]$, it follows that $R_{p} /[p(O \otimes$ $\left.\left.\mathbf{Z}_{p}\right) \cap R_{p}\right]=\mathbf{Z} / p$, and the result follows.

At this point it is necessary to recall some facts about the $e_{p}$-pairing on $E$. This pairing $e:{ }_{p} E \times{ }_{p} E \rightarrow \mu_{p}$ may be calculated as follows. Let $P_{0}$ be the origin of $E$ and let $P, Q \in{ }_{p} E$. There are functions $f, g$ on $E$ whose divisors are $p P-p P_{0}$ and $p Q-p P_{0}$ respectively. Letting $T_{R}^{*} h(S)=h(S-R)$ for any function $h$ and points $R, S \in E$, we have $e(P, Q)=f \cdot T_{P}^{*} g \cdot T_{Q}^{*} f^{-1} \cdot g^{-1}$, which is a constant. Two properties of $e(P, Q)$ are crucial. First (see Mumford [5]) the pairing is perfect, i.e., if $\zeta$ is a generator of $\mu_{p}$, there exist $P, Q \in{ }_{p} E$ such that $e(P, Q)=\zeta$. Second, let $\sigma \in G$. It is easy to see that $f^{\sigma}, g^{\sigma}$ have divisors $p \sigma P-p P_{0}$ and $p \sigma Q-p P_{0}$ respectively, and it follows that $\sigma(e(P, Q))=e(\sigma P, \sigma Q)$ for any $P, Q \in{ }_{p} E$. In particular, if $\sigma$ fixes all $p$-torsion, it likewise fixes $\mu_{p}$. Therefore $H\left(\mu_{p}\right) \subset H_{p}$.

We are in position to show that $R_{p}$ is almost always $O \otimes \mathbf{Z}_{p}$, i.e., that rationality over $H$ and stability under $O$ are equivalent for $p$-groups in $E^{g}$, for almost all $p$. 
Theorem 4. If $p$ is an odd prime, unramified in $F$, then $R_{p}=\mathbf{Z}_{p}[\alpha]$.

Proof. Let $p$ be such a prime. By the corollary, it will suffice to show that $G_{p} \nsubseteq(\mathbf{Z} / p)^{*}$. Assume the contrary. Then $\left[H_{p}: H\right] \leq p-1$. Since $F$ is unramified at $p$ and $H$ is unramified over $F, H$ is also unramified at $p$. The extension $\mathbf{Q}\left(\mu_{p}\right) / \mathbf{Q}$ has ramification degree $p-1$ at $p$. Therefore $H\left(\mu_{p}\right) / \mathbf{Q}$ likewise has ramification degree $\geq p-1$, but since $H$ is unramified at $p$ this ramification must be concentrated in $H\left(\mu_{p}\right) / H$. Thererfore $p-1 \geq\left[H_{p}: H\right] \geq$ $\left[H\left(\mu_{p}\right): H\right] \geq p-1$. Then $H_{p}=H\left(\mu_{p}\right)$ and $G_{p}=(\mathbf{Z} / p)^{*}$. Let $\sigma \in G_{p}$ act on ${ }_{p} E$ by multiplication by -1 . Since $p$ is odd, $\sigma$ is nontrivial. Let $\zeta$ generate $\mu_{p}$, and choose $P, Q$ such that $e(P, Q)=\zeta . \sigma(\zeta)=\sigma(e(P, Q))=$ $e(\sigma P, \sigma Q)=e(-P,-Q)=e(P, Q)=\zeta$, so $\sigma$ is trivial on $H\left(\mu_{p}\right)=H_{p}$. This contradiction proves the result.

Corollary. If $d$ is not a square in $\mathbf{Q}(i)$, every finite subgroup of $E_{d}^{g}$ rational over $\mathbf{Q}(i)$ is stable under $\mathbf{Z}[i]$.

Proof. Set $E=E_{d}, F=H=\mathbf{Q}(i), O=\mathbf{Z}[i] . \quad F / Q$ ramifies only at 2 . Hence, by the theorem and the remarks before the lemma, we need only show that $R_{2}=O \otimes \mathbf{Z}_{2}$, i.e., $G_{2} \nsubseteq(\mathbf{Z} / 2)^{*}=\{1\}$. But this is easy; the 2-torsion of $E_{d}$ includes the point $(\sqrt{d}, 0,1)$, which is by assumption not rational over $\mathbf{Q}(i)$. Hence $G_{2}$ is nontrivial and the result follows.

Corollary. Any Abelian variety $A$ isogenous over $\mathbf{Q}(i)$ to $E_{d}^{g}$ for nonsquare $d$ has multiplication by a map whose square is the inversion map.

Proof. Obvious.

\section{ISOGENIES AND THE NERON-SEVERI GROUP}

Recall now the Néron-Severi group $N S$ of divisors mod algebraic equivalence. For any Abelian variety $A$ there is an injection of $N S(A)$ into the second de Rham cohomology group of the complexification $A_{C}$ of $A$. Writing $A_{\mathrm{C}}=\mathrm{C}^{g} / \Lambda$ for some lattice $\Lambda$, put $z_{1}, \ldots, z_{g}$ for the coordinate functions on $\mathbf{C}^{g}$. Then $H_{D R}\left(A_{\mathbf{C}}\right)$ is generated by the forms $d z_{j} \wedge d z_{k}, d z_{j} \wedge d \bar{z}_{k}, d \bar{z}_{j} \wedge d \bar{z}_{k}$, and the image of $N S$ lies in the subspace generated by $d z_{j} \wedge d \bar{z}_{k}$ for various $j, k$. (Again, see Mumford [5].) A polarization on $A$ may be identified with an element of $N S$ containing an ample divisor. We now consider the effect of endomorphisms of $A$ on $N S$. Let $\tau \in \mathbf{C}^{*}$ act diagonally on $\mathbf{C}^{g}$. If $\tau$ stabilizes $\Lambda$, it induces an endomorphism, also written $\tau$, of $A_{\mathrm{C}}$.

Proposition. The induced endomorphism $\tau^{*}$ of $N S(A)$ acts by multiplication by $\tau \bar{\tau}$.

Proof. $\tau^{*}\left[d z_{j} \wedge d \bar{z}_{k}\right]=\left[d\left(\tau z_{j}\right) \wedge d\left(\tau z_{k}\right)^{-}\right]=\tau \bar{\tau}\left[d z_{j} \wedge d \bar{z}_{k}\right]$.

Corollary. The scalar automorphism $i$ acts trivially on $N S\left(E_{d}^{g}\right)$.

Corollary. If $\phi: A_{1} \rightarrow A_{2}$ is an isogeny, the map $\phi^{*}: N S\left(A_{2}\right) \rightarrow N S\left(A_{1}\right)$ is injective. 
Proof. Recall (Mumford [5]) that there is a map $\phi^{\prime}: A_{2} \rightarrow A_{1}$ such that $\phi \circ \phi^{\prime}$ acts on $A_{2}$ by multiplication by an integer $n$. Then $\phi^{\prime *} \circ \phi^{*}=\left(\phi \circ \phi^{\prime}\right)^{*}$ acts on $N S\left(A_{2}\right)$ by multiplication by $n^{2}$. Since $N S\left(A_{2}\right)$ is torsion-free, $\phi^{\prime *} \circ \phi^{*}$ and (hence) $\phi^{*}$ are injective.

We can now complete the proof of Theorem 2. By the remarks in the introduction, Theorem 3, and the final corollary of §III, it suffices to prove the following.

Proposition. Let $A$ be an Abelian variety, isogenous over $\mathbf{Q}(i)$ to $E_{d}^{g}$ for some nonsquare $d$. The map $i$ which descends to $A$ from $E_{d}^{g}$ stabilizes any polarization on $A$.

Proof. Let $\Theta$ be a polarization on $A$ and $\Theta_{0}=i^{*} \Theta$. Let $\phi: E_{d}^{g} \rightarrow A$ be the given isogeny. Put $D=\phi^{*} \Theta, D_{0}=\phi^{*} \Theta_{0}$. Now $D_{0}=\phi^{*} i^{*} \Theta=i^{*} \phi^{*} \Theta=$ $i^{*} D=D$ by the first corollary above. That is, $\phi^{*} \Theta=\phi^{*} \Theta_{0}$. But by the second corollary $\phi^{*}$ is injective, so $\Theta=\boldsymbol{\Theta}_{0}$ and $\Theta$ is stable under $i^{*}$. This completes the proof of Theorem 2 .

\section{THE HYPERELLIPTIC CASE}

It remains to consider the case where $C$ is hyperelliptic. The isogeny between $J$ and $E_{d}^{g}$ allows us to lift the automorphism $i$ to an automorphism $\varphi$ of $J$. Consider the action of $\varphi$ on the tangent space $T(J)$ of $J$. Since $i$ is induced by multiplication by $i$ on the tangent space of $E_{d}^{g}, \varphi$ acts likewise on $T(J)$. Now, by Theorem 3, $\varphi$ induces an automorphism $\psi$ of $C$, whose square is the hyperelliptic involution of $C$. Under the natural isomorphism between $T(J)$ and the space $\Gamma(\omega)$ of holomorphic differential forms on $C$, the action of $\varphi$ corresponds to that of $\psi$. Thus, $C$ must have an automorphism whose square is the hyperelliptic involution and which acts on holomorphic differentials by multiplication by $i$.

Now, consider the representation of $C$ as a twofold cover of $\mathbf{P}^{1}$, with equation $y^{2}=f(x)$. The automorphism $\psi$ induces an automorphism of $\mathbf{P}^{1}$, of order two; we may assume it to be the map $x \mapsto-x . f(x)$ must then be odd; $\psi$ takes the form $(x, y) \mapsto(-x, i y)$. (If $f(x)$ were even, $\psi^{2}$ would be the identity, rather than the hyperelliptic involution.) It is well known (see, e.g., Shafarevich [6]) that, under this representation, the holomorphic differentials on $C$ are spanned by the forms $x^{j} d x / y, j=0, \ldots, g-1$. But the action of the given $\psi$ on these is multiplication by $(-1)^{j} i$; it does not act as a scalar except in the trivial case $g=1$. This contradiction completes the proof of Theorem 1.

\section{ACKNOWLEDGMENT}

Part of this paper is based on the author's University of Chicago thesis; he would like to express his gratitude to Frans Oort for suggestions which helped complete the picture. 


\section{BIBLIOGRAPHY}

1. S. Bloch, Algebraic cycles and values of L-functions. I, J. Reine Angew. Math. 350 (1984), 94-108.

2. C. Ceresa, $C$ is not equivalent to $\bar{C}$ in its Jacobian, Ann. of Math. (2) 177 (1983), 285-291.

3. P. Griffiths, Periods of integrals on algebraic manifolds, Ann. of Math. (2) 90 (1968), 805-865.

4. B. Gross, Arithmetic on elliptic curves with complex multiplication, Lecture Notes in Math., vol. 776, Springer-Verlag, Berlin and New York, 1980.

5. D. Mumford, Abelian varieties, Oxford Univ. Press, London, 1970.

6. I. Shafarevich, Basic algebraic geometry, Springer-Verlag, Berlin and New York, 1974.

7. N. Stephens, Unpublished correspondence.

8. A. Weil, Zum Beweis des Torellischen Sätzes, Nachr. Akad. Wiss. Göttingen, Math. Phys. Kl. IIA, (1957), 33-53.

Department of Mathematics and Statistics, Southern Illinois University, EDWARDSVILLE, ILLINOIS 62026 